\title{
Two novel prognostic models for ovarian cancer respectively based on ferroptosis and necroptosis
}

Yang $\mathrm{Li}^{1 \dagger}$, Xiaojin Gong ${ }^{1 \dagger}$, Tongxiu Hu${ }^{1}$ and Yurong Chen ${ }^{2 *}$

\begin{abstract}
Background: Platinum-resistant cases account for $25 \%$ of ovarian cancer patients. Our aim was to construct two novel prognostic models based on gene expression data respectively from ferroptosis and necroptosis, for predicting the prognosis of advanced ovarian cancer patients with platinum treatment.

Methods: According to the different overall survivals, we screened differentially expressed genes (DEGs) from 85 ferroptosis-related and 159 necroptosis-related gene expression data in the GSE32062 cohort, to establish two ovarian cancer prognostic models based on calculating risk factors of DEGs, and log-rank test was used for statistical signifcance test of survival data. Subsequently, we validated the two models in the GSE26712 cohort and the GSE17260 cohort. In addition, we took gene enrichment and microenvironment analyses respectively using limma package and GSVA software to compare the differences between high- and low-risk ovarian cancer patients.

Results: We constructed two ovarian cancer prognostic models: a ferroptosis-related model based on eight-gene expression signature and a necroptosis-related model based on ten-gene expression signature. The two models performed well in the GSE26712 cohort, but the performance of necroptosis-related model was not well in the GSE17260 cohort. Gene enrichment and microenvironment analyses indicated that the main differences between high- and low- risk ovarian cancer patients occurred in the immune-related indexes, including the specific immune cells abundance and overall immune indexes.

Conclusion: In this study, ovarian cancer prognostic models based on ferroptosis and necroptosis have been preliminarily validated in predicting prognosis of advanced patients treated with platinum drugs. And the risk score calculated by these two models reflected immune microenvironment. Future work is needed to find out other gene signatures and clinical characteristics to affect the accuracy and applicability of the two ovarian cancer prognostic models.
\end{abstract}

Keywords: Ovarian cancer, Prognostic model, Ferroptosis, Necroptosis, Immune microenvironment

*Correspondence: chenyurongtj@163.com

†Yang Li and Xiaojin Gong contributed equally to this work.

${ }^{2}$ Department of Oncology, Zhuji People's Hospital of Zhejiang Province,

Zhuji 311800, Zhejiang, China

Full list of author information is available at the end of the article

\section{Background}

Ovarian cancer is a gynecological malignancy with the highest mortality, and ranks the fifth leading cause of cancer-related death in the USA [1]. In the USA, Ovarian cancer accounts for $2.38 \%$ of all female malignancies and $4.89 \%$ of all female cancer deaths, and the 5 -year relative survival is only $48.6 \%$ [2]. The main reason for the high mortality rate from ovarian cancer is $75 \%$ of cases 
are already at advanced stage when diagnosed [3]. But early detection of ovarian cancer is difficult due to the insidious onset, including elvictor abdominal pain, early satiety, urinary frequency, constipation and abdominal distension [4]. Similar to other cancers, metastatic disease is the main cause to ovarian cancer related deaths [5]. In ovarian cancer, platinum-taxanes combination chemotherapy is a regular treatment after surgical cytoreduction [6]. However, most patients eventually relapse due to the strong drug resistance, especially for platinum drugs [7].

Drug resistance is a major problem in cancer treatment, leading to cell tolerance and failure in response to one or multiple agents. Since $25 \%$ of ovarian cancer patients are typically platinum resistant [8], it is necessary to predict platinum efficacy to each patient before chemotherapy. According to the previous studies, platinum resistance involved many biological processes in ovarian cancer, including altered drug metabolism, role of membrane transporters, dysregulation of cellular metabolism, cell death inhibition, DNA damage repair, long non-coding RNAs, epigenetics, oxidative stress [9]. Among them, cell death inhibition is the final reason to make the ovarian cancer cell resistant to platinum [10]. Therefore, identification of ovarian cancer related factors in cell death pathways is an effective way to predict the prognosis after platinum drug.

As the main cell death type, apoptosis has been well studied in ovarian cancer, but the major drug resistance is apoptosis resistance [11]. Ferroptosis and necroptosis are two newly discovered types of regulated necrosis, and a growing number of anti-cancer drugs have been reported to function as the activators of ferroptosis or necroptosis [12-14]. Ferroptosis is an iron-catalyzed form of regulated necrosis that functions through excessive peroxidation, recent studies have found that iron ptosis plays an important role in the occurrence and development of ovarian cancer [15]. Necroptosis is defined as a regulated necrosis type that requires the receptor interacting protein kinase 3 (RIPK3) and mixed lineage kinase like (MLKL), and is induced by death-related receptors, sensors and other mediators. It has been proved that both ferroptosis and necroptosis play important roles in cancer cells, especially in drug resistance $[16,17]$. In ovarian cancer stem-like cells necroptosis was found driven by ALDH1A family selective inhibitors, which were broadly linked with resistance to chemotherapeutics such as paclitaxel and doxorubicin [18]. However, the relationship between ferroptosis- and necroptosis-related genes and prognosis of ovarian cancer patients is still vastly unknown, making it still a challenge for predicting prognosis of chemotherapy and developing novel therapies for ovarian cancer. Therefore, identifying cancer-related genes in ferroptosis and necroptosis is a promising way to predict prognosis of ovarian cancer patients using platinum drugs.

In this study, we established two prognostic models in ovarian cancer, involving ferroptosis and necroptosis respectively, based on the expression data from ovarian cancer patients with platinum drug in the GSE32062 cohort. Both two models were validated in the GSE26712 cohort, but the necroptosis-related model performed inappropriately in the GSE17260 cohort. Finally, we performed functional enrichment analysis and tumor microenvironment analysis to explore the molecular mechanisms that may influence the prognosis of ovarian cancer. Taken together, ferroptosis and necroptosis are two important pathways related to ovarian cancer prognosis, which can be used to build the prognostic prediction models under specific genetic backgrounds and platinum drug therapy.

\section{Materials and methods \\ Data collection \\ The GSE32062 cohort, the GSE26712 cohort and the GSE17260 cohort}

The ovarian cancer expression data and clinical data of GSE32062 cohort [19], GSE26712 cohort [20] and GSE17260 cohort [21] were downloaded from gene omnibus expression (GEO) database (https://www.ncbi. nlm.nih.gov/gds/). The expression data of GSE32062 cohort, GSE26712 cohort and GSE17260 cohort were produced from agilent whole human genome oligo microarray, affymetrix human genome U133A array, and Agilent-014850 whole human genome microarray $4 x 44 \mathrm{~K}$ G4112F, respectively. In GSE32062 cohort, 10 cases were excluded due to the different platform, and the other 260 ovarian cancer patients with platinum drugs were included. In the GSE26712 cohort, all 185 late-stage (III/ IV) ovarian cancer patients with platinum drugs were included as validation group 1. In GSE17260 cohort, 110 stage III/IV serious ovarian cancer patients who underwent platinum chemotherapy were included as validation group 2 .

\section{Gene set}

The ferroptosis-related gene set including 60 genes from references [22] and 41 genes from ferroptosis-related Kyoto Encyclopedia of Genes and Genomes (KEGG) pathway, among which 16 genes overlap and finally a total of 85 genes were included. All of 159 genes from necroptosis-related literatures and KEGG pathway were included as the necroptosis-related gene set in this study [23-25]. 


\section{Construction and validation prognostic models}

Based on overall survival (OS), univariate and multivariate Cox regression analysis were used to screen prognosis-related genes respectively from ferroptosisrelated gene set and necroptosis-related gene set. $P$ value $<0.1$ was the threshold for the entry from single factor into multivariate analysis, and stepwise regression was used for gene screening in multifactor analysis. The risk scores were calculated according to the normalized expression level of each screened gene and its corresponding regression coefficients. Patients were divided into high and low risk groups based on the median value of the risk score. Survival (version: 3.2-3) package and SurvMiner (version: 0.4.8) package were used for drawing survival curve, and log-rank test method was used for statistical significance test of survival data.

\section{Enrichment analysis}

Differentially expressed genes (DEGs) were screened between high and low risk groups by limma package, and the screening threshold was $\left|\log _{2} \mathrm{FC}\right|>2$ and $P$ value $<0.05$. The screened DEGs were enriched in gene ontology (GO) and KEGG pathways by DAVID (https://david. ncifcrf.gov/).

\section{Immune microenvironment analysis}

Relative abundance of each immune cell was calculated by GSVA (version: 1.32.0) package. Immune infiltration related indexes were calculated by ESTIMATE, including immune score, stromal score and ESTIMATE score. Correlation analysis between immune-related indexes and ferroptosis/necroptosis-related genes was conducted using psych (version: 2.0.8) package and drawn by corrplot (version: $0.84)$ package. Differential immune indexes between high and low risk groups were analyzed and drawn using stats (version: 3.6.2) package and ggpubr (version: 0.4.0) package.

\section{Statistical analysis}

Student's t-test was used to compare gene expression and immune related indexes between high and low risk groups. Log-rank test was used to compare OS between different groups by Kaplan-Meier analysis. Univariate and multivariate Cox regression analyses were applied using survival (version: 3.2-3) and glmnet (version: 4.0-2) packages in $\mathrm{R}, P<0.1$ was the threshold. All statistical analyses were conducted in $\mathrm{R}$ software. All $P$ values were two-tailed, and less than 0.05 was considered statistically significant.

\section{Results}

The work flow of this study is shown in Fig. 1. First, a total of 260 stage III/IV ovarian cancer patients with platinum treatment from GSE32062 were enrolled to construct a Cox model to predict the prognosis. Second, a total of 185 and 110 stage III/IV ovarian cancer patients with platinum treatment respectively from GSE26712 and GSE17260 were used as the validation group (validation group 1 and 2) to verify the models. The basic clinical characteristics of enrolled patients are listed in Table 1.

\section{Identification of the prognostic ferroptosis-related} and necroptosis-related genes in the GSE32062 cohort

A total of 85 ferroptosis-related genes and a total of 159 necroptosis-related genes were used to analyze the relationship with prognosis of ovarian cancer patients in the GSE32062 cohort, respectively. Based on the expression profile, through the univariate and multivariate Cox regression analyses with OS, eight DEGs related to ferroptosis were finally identified closely related to OS, including NFS1, ATG7, G6PD, VDAC2, SLC3A2, $M A P 1 L C 3 C, A C S L 3$, and PTGS2 (Fig. 2a). Through the same method, we screened 10 necroptosis-related DEGs associated with OS of ovarian cancer patients, namely STAT5B, CAMK2D, HIST1H2AJ, CASP1, PYGB, IFNAR2, CAMK2G, STAT1, FADD, and HMGB1 (Fig. 2b). The functions and ovarian cancer-related research of these genes are listed in Supplementary Table S1.

\section{Construction of four ferroptosis-related and necroptosis-related prognostic models in the GSE32062 cohort}

Based on the identified DEGs, we constructed two prognostic models for ovarian cancer through Cox regression analyses. The risk score was calculated by the DEGs $\left(\mathrm{n}_{\text {(ferroptosis) }}=8, \mathrm{n}_{\text {(necroptosis) }}=10\right)$, and the Yoden index was used to calculate the best cutoff value to divide the enrolled ovarian cancer patients into high and low risk groups (Fig. 2c, d). The characteristics of two models were described as follows.

\section{Ferroptosis-related prognostic model}

We used eight screened DEGs to construct the ferroptosis-related prognostic model with OS. The OS of ovarian cancer patients prolonged with the high-expression of ATG7, G6PD, SLC3A2, MAP1LC3C and PTGS2, but shrank with the increased expression of NFS1, VDAC2, ACSL3 (Fig. 2a). According to the best cutoff value of risk score, we divided 260 enrolled ovarian cancer patients into high-risk group $(n=116)$ and low-risk group 


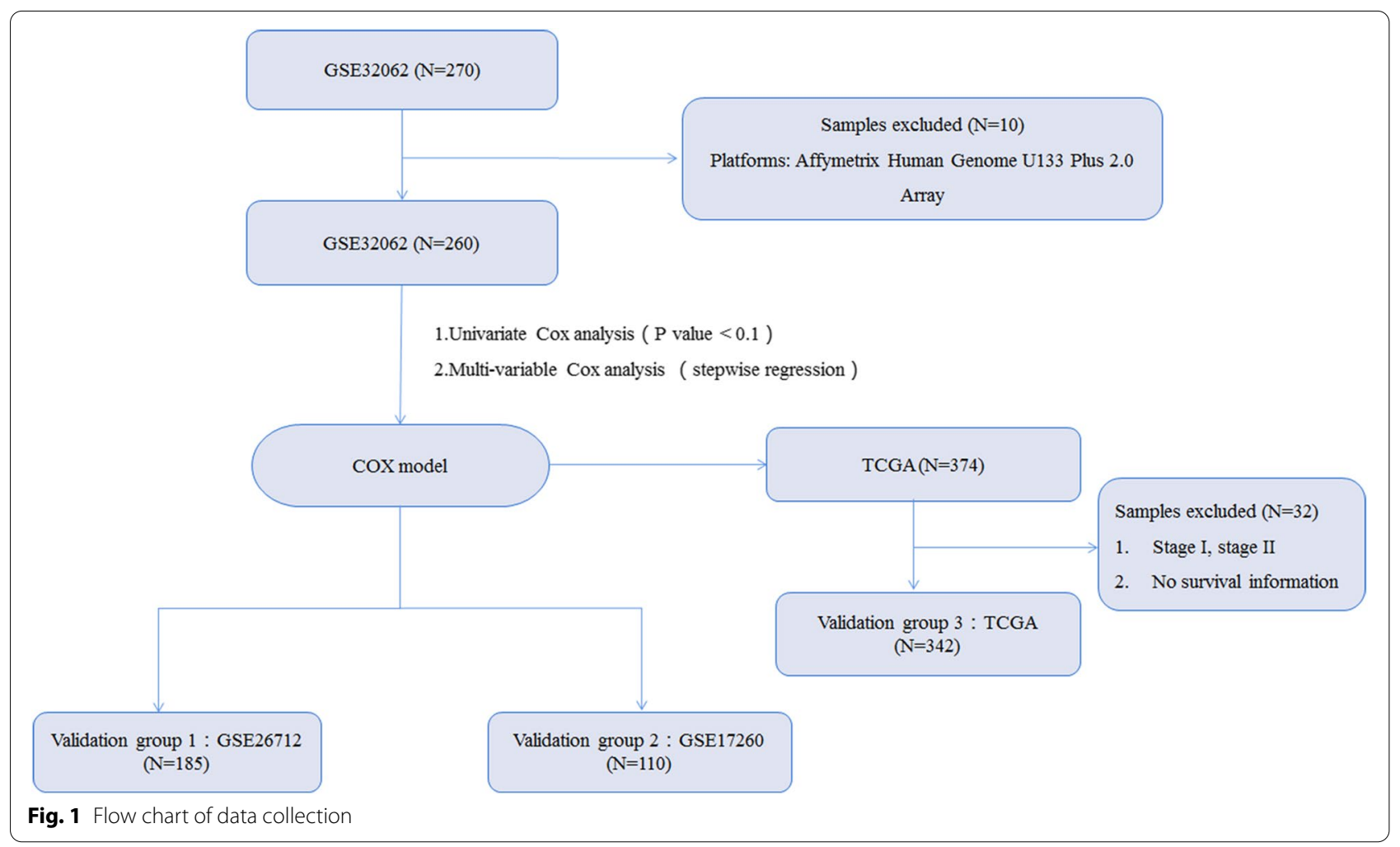

$(n=144)$, shown in Fig. 2c. Survival analysis showed that the OS of high-risk group was significantly shorter than low-risk group $(P<0.0001 ;$ Fig. 3a). The risk score for OS was calculated by the predictive performance of the eight-gene receiver operating characteristic (ROC) curves. The area under the curve (AUC) reached 0.632 of 3-year survival, 0.683 of 5-year survival, and 0.681 of 10-year survival (Fig. 3b).

\section{Necroptosis-related prognostic model}

To build the necroptosis-related OS prognostic model, we used ten selected DEGs of STAT5B, CAMK2D,

Table 1 Clinical characteristics of patients.

\begin{tabular}{|c|c|c|c|c|}
\hline Cohort & & GSE32062 & GSE26712 & GSE17260 \\
\hline Total number & & 260 & 185 & 110 \\
\hline Age (mean $\pm S D)$ & & $58.2 \pm 10.8$ & $62 \pm 12$ & $59 \pm 12.6$ \\
\hline \multirow[t]{2}{*}{ Histology } & Serous & $260(100 \%)$ & $166(89.73 \%)$ & $110(100 \%)$ \\
\hline & Others & 0 & $19(10.27 \%)$ & 0 \\
\hline \multirow[t]{4}{*}{ Stage } & Stage I & 0 & 0 & 0 \\
\hline & Stage II & 0 & 0 & 0 \\
\hline & Stage III & $204(78.46 \%)$ & $144(77.84 \%)$ & 93 (84.55\%) \\
\hline & Stage IV & $56(21.54 \%)$ & $41(22.16 \%)$ & $17(15.45 \%)$ \\
\hline \multirow[t]{4}{*}{ Grade } & Grade 1 & 0 & 0 & $26(23.64 \%)$ \\
\hline & Grade 2 & $131(50.38 \%)$ & $40(21.62 \%)$ & $41(37.27 \%)$ \\
\hline & Grade 3 & $129(49.62 \%)$ & $144(77.84 \%)$ & $43(39.09 \%)$ \\
\hline & Grade 4 & 0 & $1(0.54 \%)$ & 0 \\
\hline \multirow[t]{2}{*}{ Treatment } & Platinum & $260(100 \%)$ & $185(100 \%)$ & $110(100 \%)$ \\
\hline & Taxane & $260(100 \%)$ & 185 (100\%) & $110(100 \%)$ \\
\hline OS (median (interquartiles)) & & $\begin{array}{l}41.5(1128) \\
\text { months }\end{array}$ & $\begin{array}{l}3.19(0.06,13.65) \\
\text { years }\end{array}$ & $\begin{array}{l}30.5(1,81) \\
\text { months }\end{array}$ \\
\hline
\end{tabular}

OS overall survival, $S D$ standard deviation 


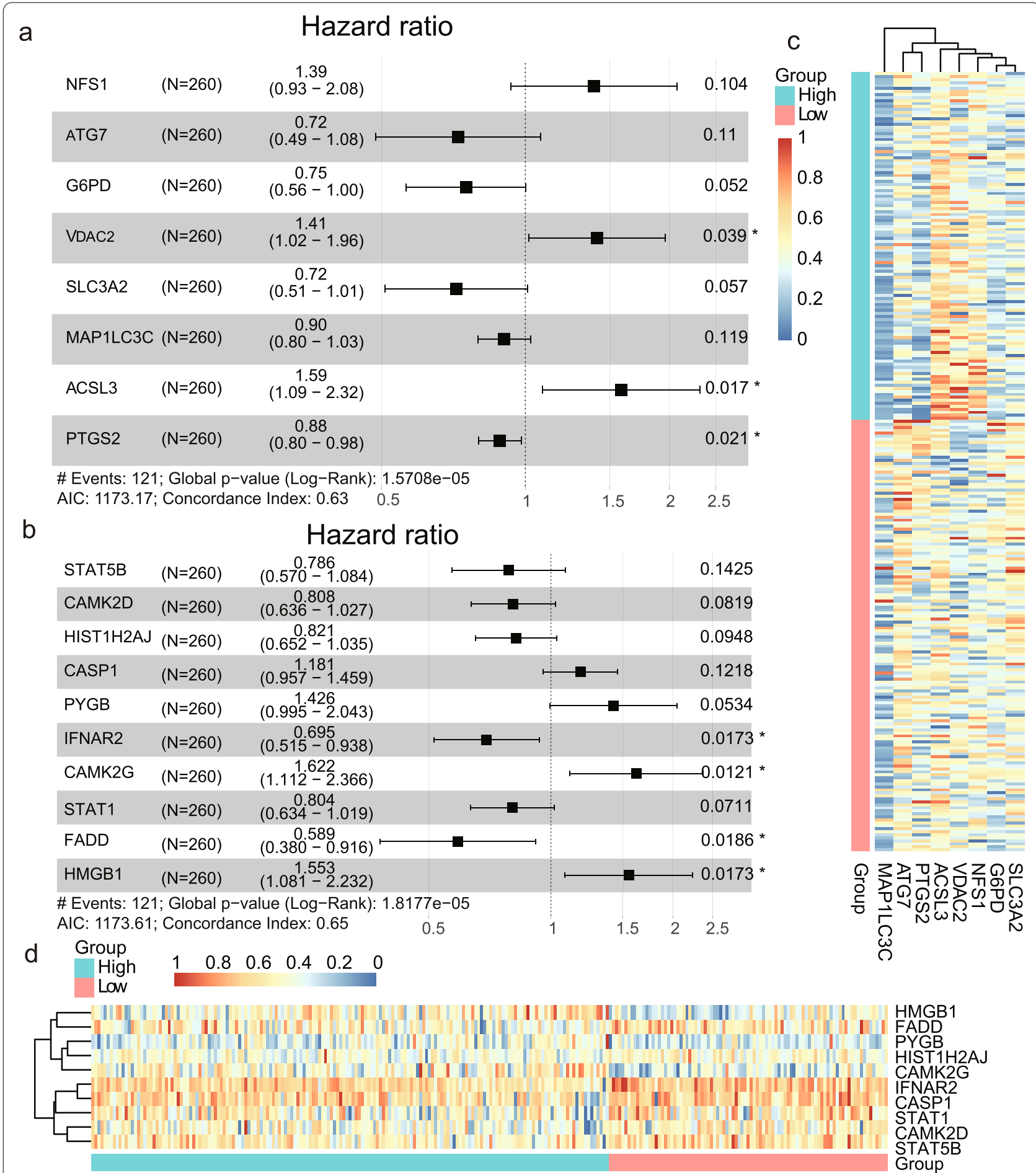

Fig. 2 Prognostic models for ovarian cancer constructed in GSE32062. a Hazard ratio of genes included in the ferroptosis-related prognostic model. b Hazard ratio of genes included in the necroptosis-related prognostic model. $\mathbf{c}$ Heatmap of gene expression in the ferroptosis-related prognostic model. $\mathbf{d}$ Heatmap of gene expression in the necroptosis-related prognostic model 


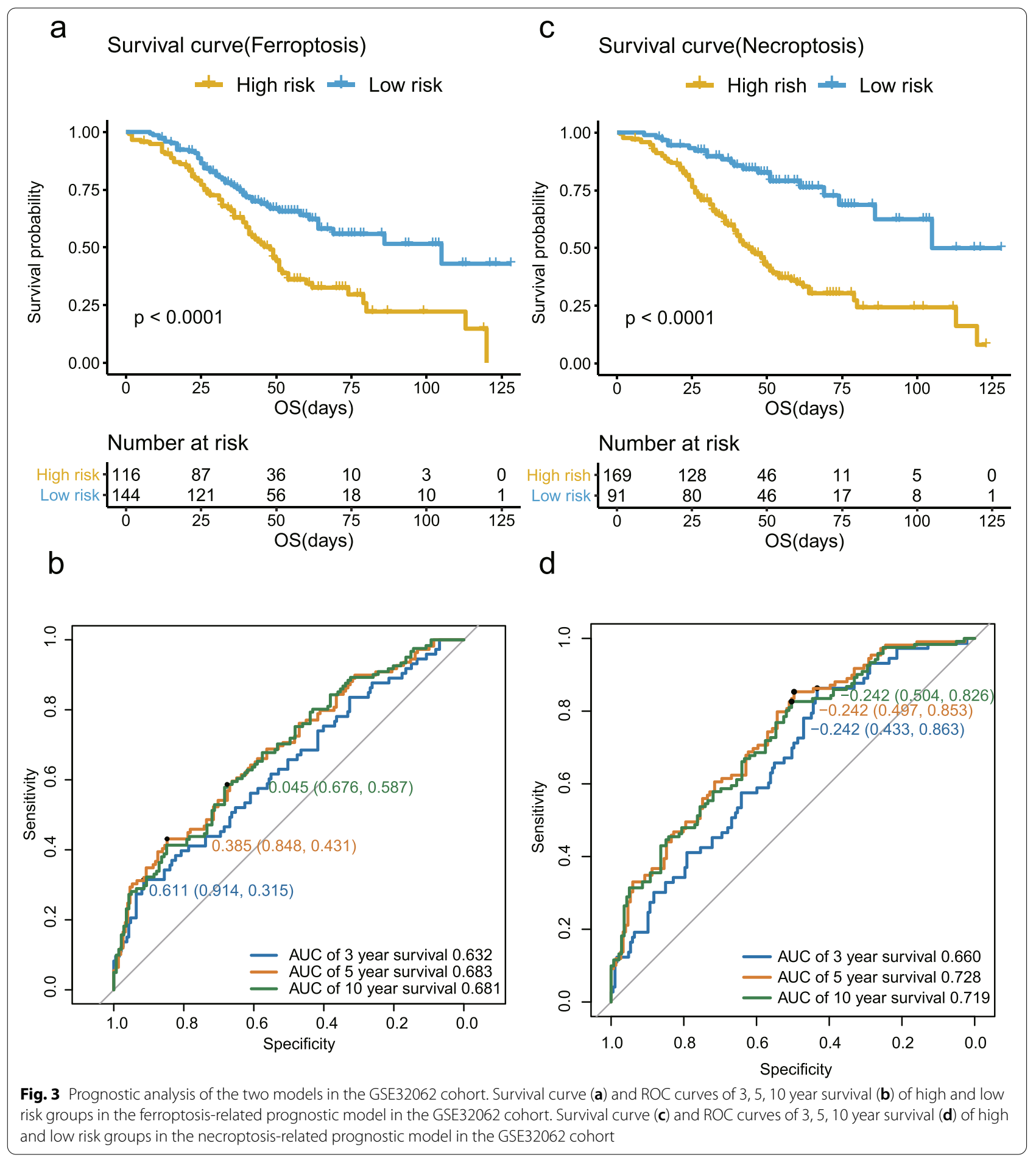

HIST1H2AJ, CASP1, PYGB, IFNAR2, CAMK2G, STAT1, FADD and HMGB1.The DEGs of STAT5B, CAMK2D, HIST1H2AJ, IFNAR2, STAT1 and FADD were identified as six protective factors to this model, and CASP1, PYGB, CAMK2G, and HMGB1 were identified as four risk factors (Fig. 2b). Survival analysis also showed a low-risk group was much longer than high-risk group in OS $(P<0.0001$; Fig. 3c). The AUC of the ten-gene ROC curves reached 0.660 of 3 -year survival, 0.728 of 5 -year survival, and 0.719 of 10 -year survival (Fig. 3d). 

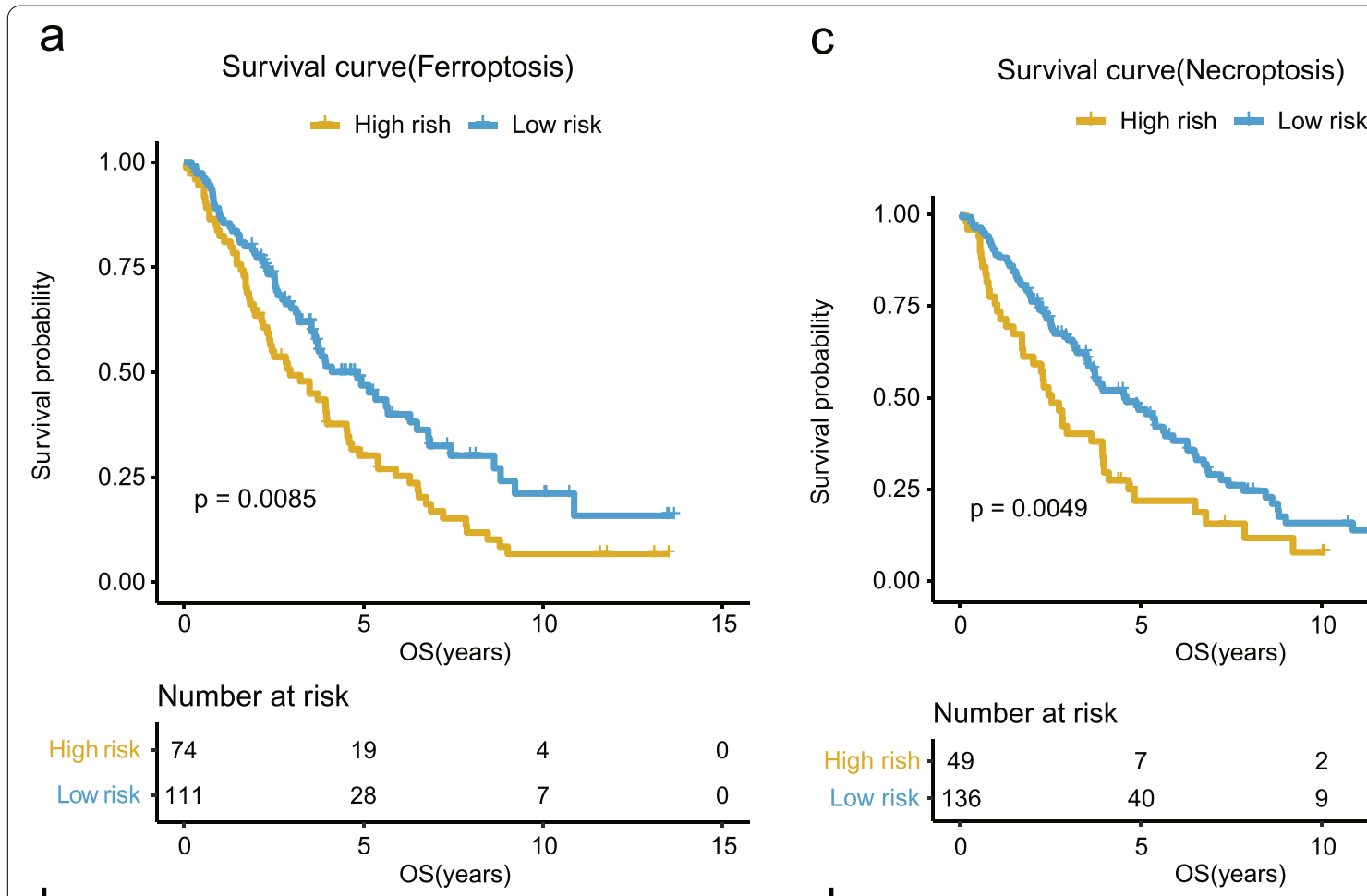

b
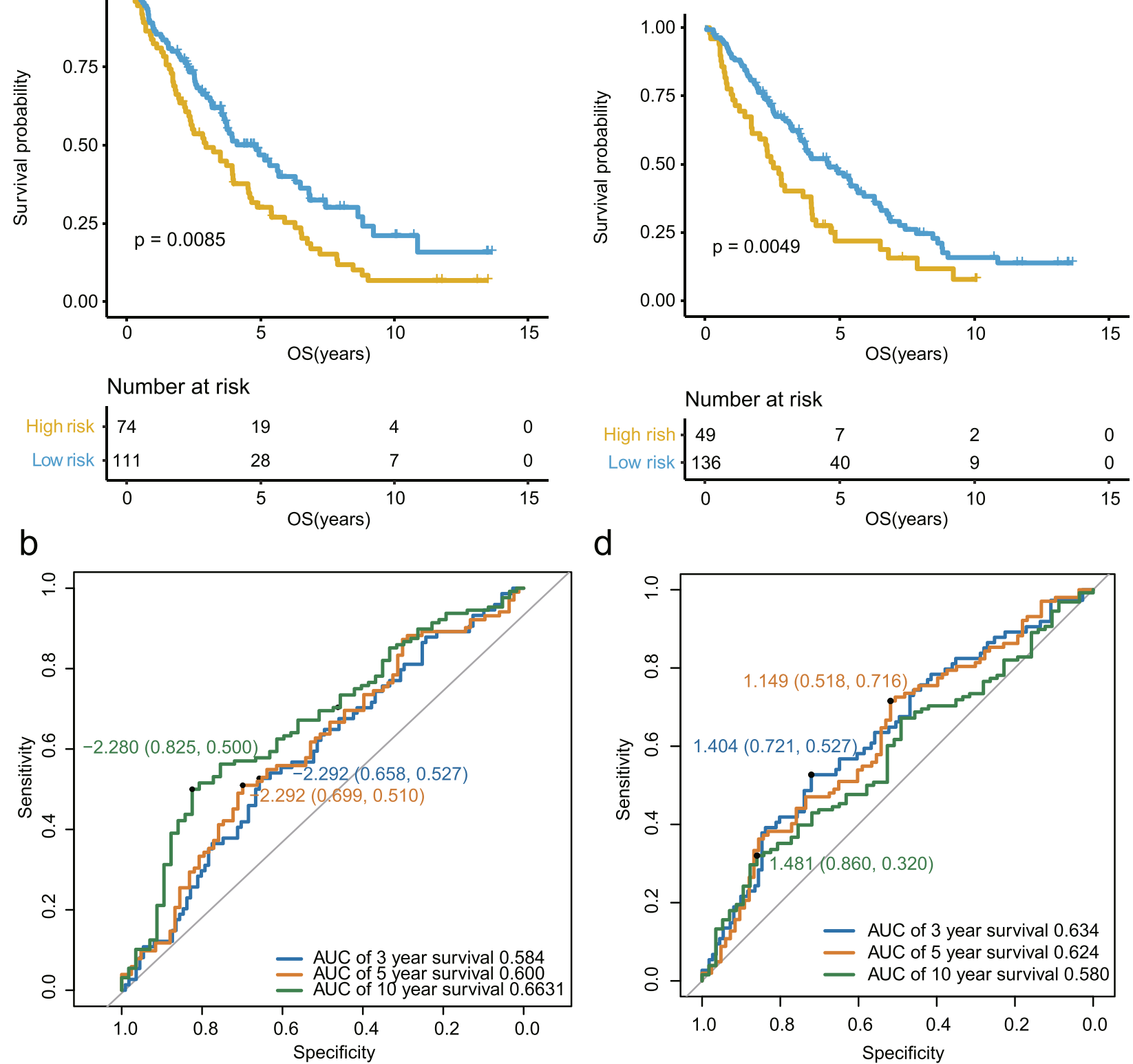

d

\begin{tabular}{|c|c|c|c|c|}
\hline High rish & 49 & 7 & 2 & 0 \\
\hline Low risk & 136 & 40 & 9 & 0 \\
\hline & 0 & 5 & 10 & 15 \\
\hline
\end{tabular}

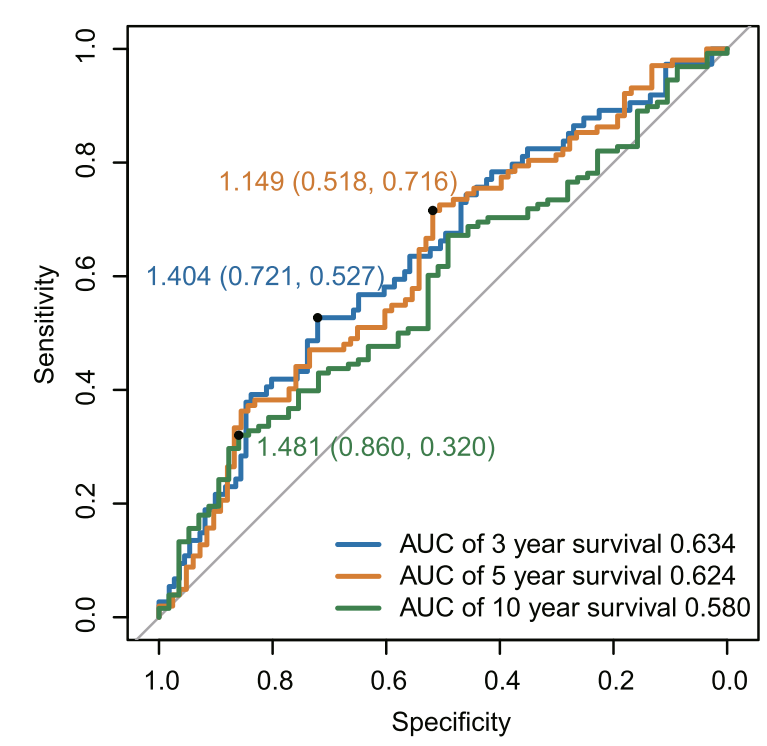

Fig. 4 Performance of the two models in the GSE26712 cohort. Survival curve (a) and ROC curves (b) of high and low risk groups in the ferroptosis-related prognostic model in the GSE26712 cohort. Survival curve (c) and ROC curves (d) of high and low risk groups in the necroptosis-related prognostic model in the GSE26712 cohort

\section{Validation of the prognostic models in the GSE26712} cohort

Here, we used GSE26712 cohort to validate the ferroptosis-related and necroptosis-related prognostic models with OS. The AUC of ferroptosis-related ROC was 0.584 of 3-year survival, 0.600 of 5-year survival, 0.663 of 10-year survival (Fig. 4b). And the AUC of necroptosisrelated ROC was 0.634 of 3-year survival, 0.624 of 5 -year survival, 0.580 of 10-year survival (Fig. 4d).

Following the same formula of risk score from GSE32062 cohort, we calculated risk score of each patient enrolled from the GSE26712 cohort. According 


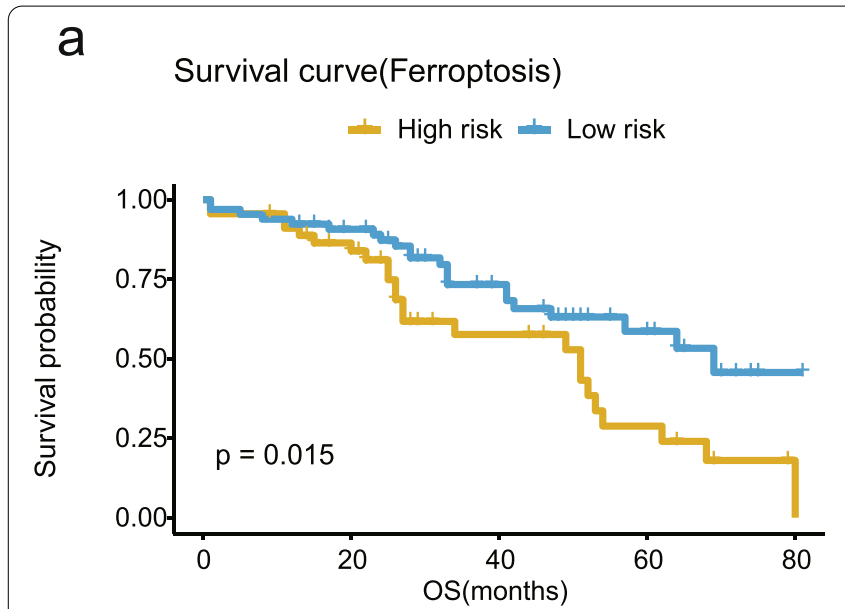

C

\section{Survival curve(Necroptosis)}

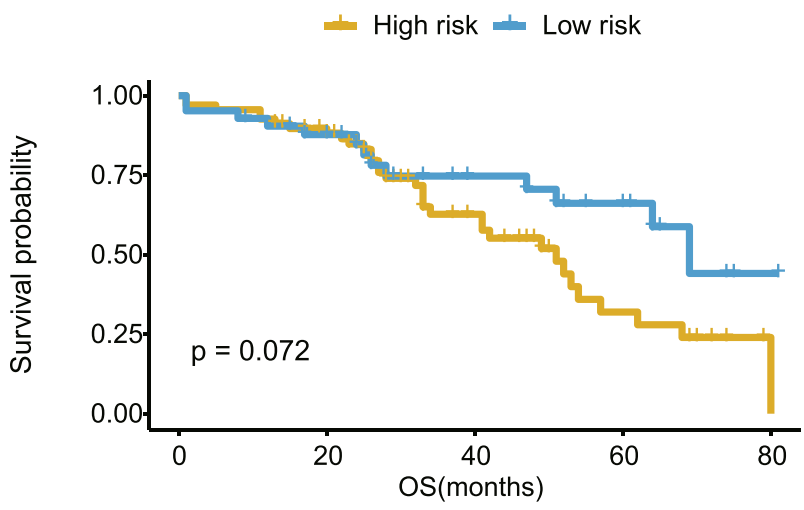

Number at risk

High risk
Low risk \begin{tabular}{ccccc}
68 & 57 & 25 & 8 & 1 \\
42 & 31 & 18 & 11 & 1 \\
\hline 0 & 20 & 40 & 60 & 80 \\
& & & OS(months)
\end{tabular}

d

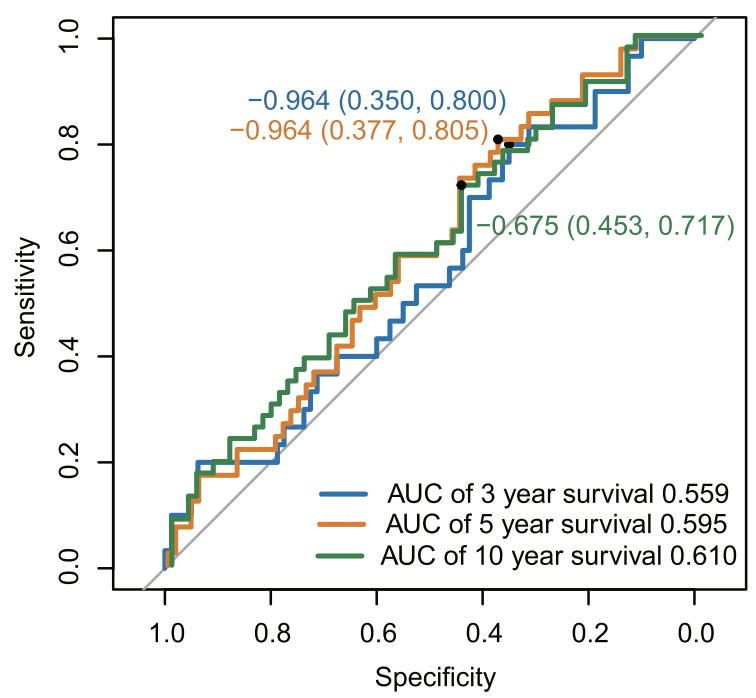

Fig. 5 Performance of the two models in the GSE17260 cohort. Survival curve (a) and ROC curves (b) of high and low risk groups in the ferroptosis-related prognostic model in the GSE17260 cohort. Survival curve (c) and ROC curves (d) of high and low risk groups in the necroptosis-related prognostic model in the GSE17260 cohort

to the risk scores, the GSE26712 cohort were divided into high-risk group and low-risk group. The OS of high-risk group was significantly shorter than lowrisk group in ferroptosis-related prognostic model $(P=0.0085$; Fig. $4 \mathrm{a})$, and the similar result was also shown in necroptosis-related prognostic model $(P=0.0049$; Fig. 4c). Therefore, the ferroptosis- and the necroptosis-related prognostic models with OS for ovarian cancer also worked well in the GSE26712 cohort.

\section{Validation of the prognostic models in the GSE17260 cohort}

To ensure the robustness of the two models in our study, a total of 110 ovarian cancer patients from the GSE17260 cohort were enrolled for further validation. The patients were clustered into high-risk group and low-risk group 
following the same way used in the GSE32062 cohort. The AUC of 3-, 5- and 10-year survival were 0.573, 0.588 and 0.594 in ferroptosis-related ROC (Fig. 5b), and 0.559, $0.595,0.610$ respectively in necroptosis-related ROC (Fig. 5d). Meanwhile, the survival curve showed significant differences between high and low risk groups in ferroptosis-related model $(P=0.015$; Fig. $5 \mathrm{a})$, but no statistical significance in the necroptosis-related model $(P=0.072$; Fig. $5 \mathrm{c})$. The results revealed that the ferroptosis-related prognostic model with OS for ovarian cancer worked well in the GSE17260 cohort, but the necroptosis-related model should be further optimized in the future.

\section{Functional enrichment analysis in the GSE32062 cohort}

To explore the biological functions and regulatory pathways related to the prognostic models, we selected the DEGs between the high-risk group and low-risk group to conduct GO and KEGG analyses. Interestingly, many immune related functions and pathways were enriched in both ferroptosis-related and necroptosis-related risk models (Fig. 6a, d).

In the ferroptosis-related prognostic model, 10 immune-related $\mathrm{GO}$ terms were significantly enriched in $(P<0.05)$, including five biological processes $(\mathrm{BPs})$ of immune response, humoral immune response, innate immune response, chemokine-mediated signaling pathway, positive regulation of NF- $\mathrm{KB}$ transcription factor activity; and five immune-related molecular functions (MFs) of chemokine activity, cytokine activity, Toll-like receptor 4 binding, CCR chemokine receptor binding and CXCR chemokine receptor binding (Fig. 6b). Besides, six immune-related KEGG pathways were also enriched in the ferroptosis-related prognostic model, including cytokine-cytokine receptor interaction, chemokine signaling pathway, TNF signaling pathway, NF-kappa B signaling pathway, NOD-like receptor signaling pathway and Toll-like receptor signaling pathway (Fig. 6c).

In the necroptosis-related prognostic model, two immunerelated BPs were enriched, including chemokine-mediated signaling pathway and immune response; three immunerelated MFs were enriched, including chemokine activity, CCR chemokine receptor binding and CXCR3 chemokine receptor binding; two immune-related KEGG pathways were enriched, including chemokine signaling pathway and cytokine-cytokine receptor interaction (Fig. 6e, f).

\section{Microenvironment analysis in the GSE32062 cohort}

According to the functional enrichment analysis, immune-related factors were identified as key elements to ovarian cancer patients in the GSE32062 cohort. To comprehensively analyze the importance of the immune-related factors in the two above prognostic models, 45 immune-related indexes calculated from the gene expression data were included.

In the ferroptosis-related prognostic model, 43 of the 45 immune-related factors had significant relationship with risk score, and plasma cell and $\mathrm{mDC}$ were the two exceptions. As expected, almost all the immune-related factors had negative relationships with risk scores, except activated CD4 and activated CD8 (Fig. 7a). For the 10 DEGs enrolled in the ferroptosis-related prognostic model, we comprehensively analyzed the correlation between each DEG expression and immune-related factors. NFS1, ATG7, VDAC2 and PTGS2 were four genes associated with more than five factors. NFS1 and VDAC2 were two DEGs that were negatively associated with immunerelated factors, involving 9 and 5 factors, respectively. ATG7 and PTGS2 were positively associated with 13 and 21 immune-related factors, respectively (Fig. 7b).

In the necroptosis-related prognostic model, 35 of 45 immune-related factors had significant relationship with risk score, and all of them showed positive correlation $(P<0.05$; Fig. $7 \mathrm{a})$. For the $12 \mathrm{DEGs}$ enrolled in this model, except CAMK2D-NK56 dim and IFMAR2activated $\mathrm{CD} 8$, all the significant relationships between each gene and factors were positive. For the correlation analysis of each DEG with immune-related factors, five DEGs were associated with more than 30 immunerelated factors, including CASP1, IFNAR2, STAT1, CYLD and STAT2. Among them, CASP1 was a DEG most associated with immune-related factors, involving 40 factors. On the contrary, five DEGs were associated with less than 2 immune-related factors, including HIST1H2AJ, PYGB, CAMK2G, FADD and HMGB1. $C A M K 2 G$ was a DEG associated with none of the 45 immune-related factors (Fig. 7c).

\section{Discussion}

In this study, we constructed two ovarian cancer prognostic models based on the GSE32062 cohort, related to ferroptosis and necroptosis, respectively. The ferroptosisrelated prognostic model performed well in both of the two validation groups, but the necroptosis-related model was validated in only one cohort (Figs. 4, 5). Both functional analysis and microenvironment analysis showed that immune-related factors had the close relationship with the risk score in the two prognostic models.

Although the current standard treatment is primary surgery followed by platinum-based chemotherapy, there is still a significant proportion of patients with platinumresistant ovarian cancer [26]. Recent studies reported that ferroptosis and necroptosis are two important pathways to affect the efficacy of platinum drugs [12-14], 


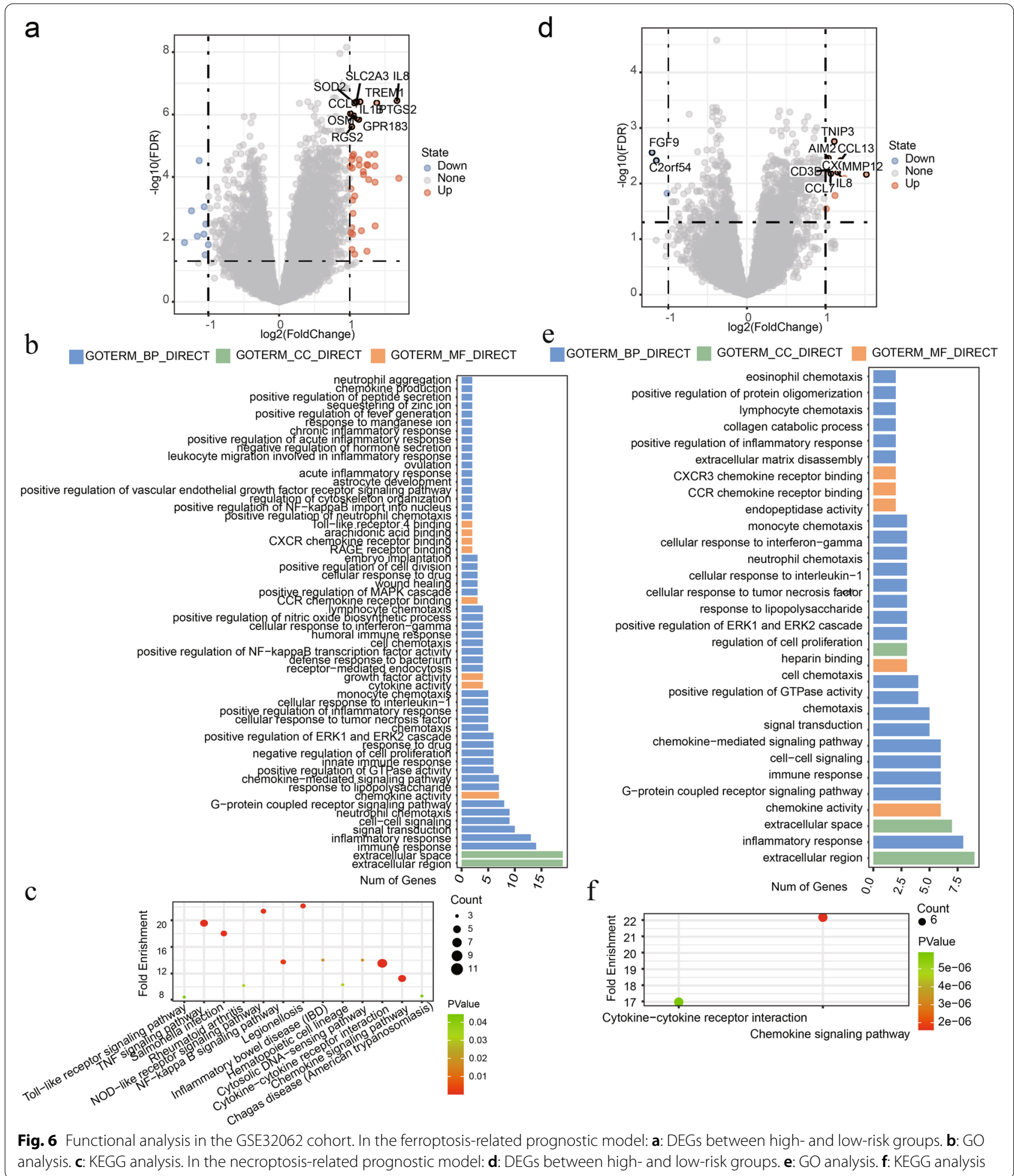

which may further affect the prognosis of ovarian cancer patients treated with platinum drugs. In this study, we established two ovarian cancer prognostic models based on gene expression data respectively of ferroptosis and necroptosis pathways, and these two prognostic models performed well in both the modeling cohort of GSE32062 


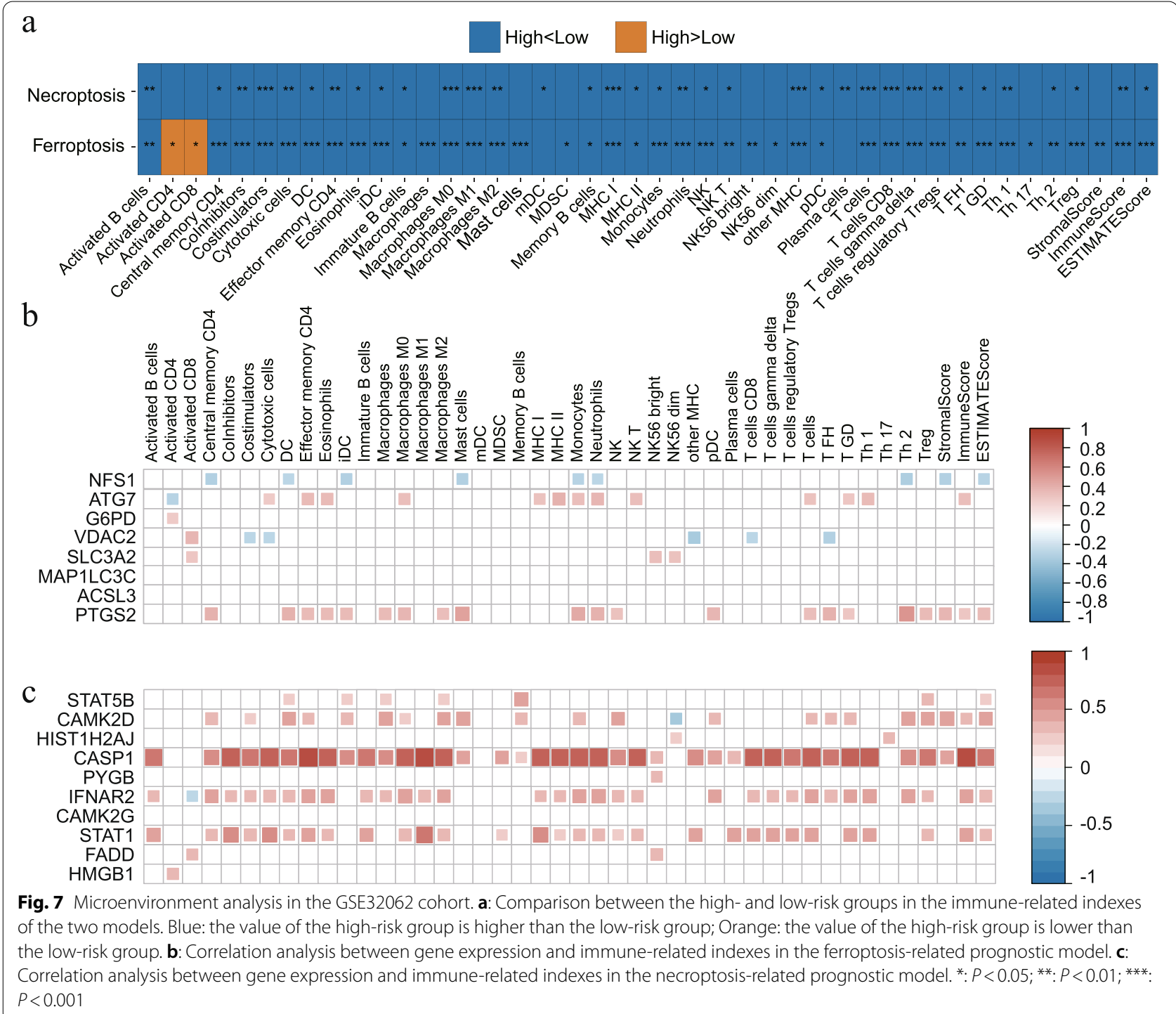

and the validation cohort of GSE26712 (Figs. 3, 4), and the ferroptosis-related model performed well in the GSE17260 cohort (Fig. 5a-b).

Besides, although the necroptosis-related prognostic model cannot be applied in the GSE17260 cohort, the survival probability after 36 months were obviously different between high and low risk groups (Fig. 5c). These results suggested that some clinical indicators, as well as expression levels of genes apart from the gene set used in this study, need to be taken into consideration. Studies have found that some clinical characteristics, such as age, performance status, FIGO stage, residual disease, histology, and BRCA/HRD, are the potential prognostic factors of ovarian cancer [27-29]. The model constructed by neglecting these factors may not be suitable for some patients. However, due to the use of common data from GEO database, these cases lack many clinical indicators and cannot be used for multivariate analysis, which is also a shortcoming of this study. The models should be further optimized based on prospective clinical study to improve their clinical usefulness.

As immune infiltrate in the microenvironment plays a key role in ovarian cancer development [30], combined with the enrichment results of many immune-related genes in the current study, we conducted a comprehensive correlation analysis to find out which immune cells were involved in the two prognostic models. The risk scores of the two ovarian cancer prognostic models had the negative correlation with ESTIMATE score, immune score and stromal score, as well as with the relative abundance 
of most immune cells (Fig. 7a). Among them, activated CD4 and activated CD8 were only two types of immune cells that could promote the ovarian cancer progress in the ferroptosis-related pathway, and similar results were also reported in the previous studies [31,32]. However, in the necroptosis-based prognostic model, all involved immunerelated indexes in this study showed a negative relationship with risk scores. These results indicated that the abundance of activated CD4 and activated CD8 could be used as a key biomarker to distinguish whether ferroptosis or necroptosis plays a dominant role in an ovarian cancer patient.

Although we display a series of significant results, there are still some limitations in this study. First, the included genes of ferroptosis and necroptosis in this study are mainly based on previous studies, so that some unreported related genes may be ignored and excluded. This problem would reduce the accuracy and applicability of the prognostic models. Second, the lack of clinical information to build the prognostic models may miss by key prognostic factors. Third, the two prognostic models constructed for ovarian cancer patients in this study need to verification in the clinical practice. According to the above, future work will focus on the two points: (1) Screening novel genes related to ferroptosis and necroptosis in more cohorts; (2) Collecting adequate ovarian cancer cases and clinical information to validate and optimize the two prognostic models, and compare them with the clinical gold standard, so as to make sure that the prognosis models are useful for clinical practice.

\section{Conclusions}

In conclusion, based on ferroptosis and necroptosis, our study constructed two ovarian cancer prognostic models for predicting the prognosis of advanced ovarian cancer patients treated with platinum drugs. The two models proved closely related to tumor immunity. Further studies are needed to optimize the two models by enrolling in other related genes and clinical information.

\section{Abbreviations}

RIPK3: Receptor interacting protein kinase 3; MLKL: Mixed lineage kinase like; KEGG: Kyoto Encyclopedia of Genes and Genomes; OS: Overall survival; DEGs: Differentially expressed genes; GO: Gene ontology; ROC: Receiver operating characteristic; AUC: Area under the curve; BPs: Biological processes; MFs: Molecular functions

\section{Supplementary Information}

The online version contains supplementary material available at https://doi. org/10.1186/s12885-021-09166-9.

Additional file 1: Supplementary Table S1. Main functions and researches of genes identified in the prognosis signatures $\#$.
Acknowledgements

Not applicable.

\section{Authors' contributions}

YR C: Concepts, Design and Manuscript editing. Y L: Data acquisition and Manuscript Preparation. XJ G and TX H: Data analysis and Statistical analysis. All authors participated in preparing the manuscript and approved the final submitted.

Funding

No funding.

\section{Availability of data and materials}

The datasets generated and/or analyzed during the current study are available in the GEO repository. (https://www.ncbi.nlm.nih.gov/gds/).

\section{Declarations}

Ethics approval

The study was approved by Tianjin Hospital.

\section{Consent for publication}

Not applicable.

\section{Competing interests}

The authors declare that they have no competing interests.

\section{Author details}

${ }^{1}$ Department of Obstetrics and Gynecology, Tianjin Hospital, Tianjin 300211 , China. ${ }^{2}$ Department of Oncology, Zhuji People's Hospital of Zhejiang Province, Zhuji 311800, Zhejiang, China.

Received: 26 March 2021 Accepted: 30 December 2021

Published online: 17 January 2022

References

1. Siegel RL, Miller KD, Jemal A. Cancer statistics, 2020. CA Cancer J Clin. 2020:70(1):7-30

2. Howlader N, Noone AM, Krapcho M, Miller D, Brest A, Yu M, Ruhl J, Tatalovich Z, Mariotto A, Lewis DR, Chen HS, Feuer EJ, Cronin KA (eds). SEER Cancer Statistics Review, 1975-2017, National Cancer Institute. Bethesda, MD, https://seer.cancer.gov/csr/1975_2017/, based on November 2019 SEER data submission, posted to the SEER web site. 2020 .

3. Winter WE III, Maxwell GL, Tian C, Sundborg MJ, Rose GS, Rose PG, et al. Tumor residual after surgical cytoreduction inprediction of clinical outcome in stage IV epithelial ovariancancer: a gynecologic oncology group study. J Clin Oncol. 2008;26(1):83-9.

4. Song YS, Kim HS, Aoki D, Dhanasekaran DN, Tsang BK. Ovarian cancer. Biomed Res Int. 2014;2014:764323.

5. Nimmagadda S, Penet MF. Ovarian cancer targeted theranostics. Front. Oncol. 2020;9:1537

6. Kaye S. Management of partially platinum-sensitive relapsedovarian cancer. Eur J Cancer. 2008;6(Suppl):16-21.

7. Davis A, Tinker AV, Friedlander M. "Platinum resistant" ovarian cancer: what is it, who to treat and how to measure benefit? Gynecol Oncol. 2014;133(3):624-31.

8. Ushijima K. Treatment for recurrent ovarian cancer-at first relapse. J Oncol. 2009:2010:497429.

9. Norouzi-Barough L, Sarookhani MR, Sharifi M, Moghbelinejad S, Jang joo S, Salehi R. Molecular mechanisms of drug resistance in ovarian cancer. J Cell Physiol. 2018;233(6):4546-62.

10. Dilruba S, Kalayda GV. Platinum-based drugs: past, present and future. Cancer Chemother Pharmacol. 2016;7(6):1103-24.

11. Binju M, Amaya-Padilla MA, Wan G, Gunosewoyo H, Rahmanto YS, Yu $Y$. Therapeutic inducers of apoptosis in ovarian cancer. Cancers (Basel). 2019;11(11):1786. 
12. Conrad M, Angeli JP, Vandenabeele P, Stockwell BR. Regulated necrosis: disease relevance and therapeutic opportunities. Nat Rev Drug Discov. 2016;15(5):348-66.

13. Fulda S. Therapeutic exploitation of necroptosis for cancer therapy. Semin Cell Dev Biol. 2014;35:51-6.

14. Yang WS, Stockwell BR. Synthetic lethal screening identifies compounds activating iron-dependent, nonapoptotic cell death in oncogenic-RASharboring cancer cells. Chem Biol. 2008;15(3):234-45.

15. Yang L, Tian S, Chen Y, Miao C, Zhao Y, Wang R, et al. Ferroptosis-related gene model to predict overall survival of ovarian carcinoma. J Oncol. 2021;2021:6687391.

16. Bebber CM, Müller F, Prieto Clemente L, Weber J, von Karstedt S. Ferroptosis in cancer cell biology. Cancers. 2020;12(1):164.

17. Gong Y, Fan Z, Luo G, Yang C, Huang Q, Fan K, et al. The role of necroptosis in cancer biology and therapy. Mol Cancer. 2019;18(1):100.

18. Chefetz I, Grimley E, Yang K, Hong L, Vinogradova EV, Suciu R, et al. A PanALDH1A inhibitor induces necroptosis in ovarian cancer stem-like cells. Cell Rep. 2019;26(11):3061-3075.e6.

19. Yoshihara K, Tsunoda T, Shigemizu D, Fujiwara H, Hatae M, Fujiwara H, et al. High-risk ovarian cancer based on 126-gene expression signature is uniquely characterized by downregulation of antigen presentation pathway. Clin Cancer Res. 2012;18(5):1374-85.

20. Bonome T, Levine DA, Shih J, Randonovich M, Pise-Masison CA, Bogomolniy $F$, et al. A gene signature predicting for survival in suboptimally debulked patients with ovarian cancer. Cancer Res. 2008;68(13):5478-86.

21. Yoshihara K, Tajima A, Yahata T, Kodama S, Kodama S, Fujiwara H, et al. Gene expression profile for predicting survival in advanced-stage serous ovarian cancer across two independent datasets. PLoS One. 2010;5(3):e9615.

22. Liang JY, Wang DS, Lin HC, Chen XX, Yang H, Zheng Y, et al. A novel ferroptosis-related gene signature for overall survival prediction in patients with hepatocellular carcinoma. Int J Biol Sci. 2020;16(13):2430-41.

23. Kanehisa M, Goto S. KEGG: Kyoto encyclopedia of genes and genomes. Nucleic Acids Res. 2000;28(1):27-30.

24. Kanehisa M. Toward understanding the origin and evolution of cellular organisms. Protein Sci. 2019;28(11):1947-51.

25. Kanehisa M, Furumichi $M$, Sato $Y$, Ishiguro-Watanabe $M$, Tanabe $M$. KEGG: integrating viruses and cellular organisms. Nucleic Acids Res. 2021:49(D1):D545-51.

26. Oronsky B, Ray CM, Spira Al, Trepel JB, Carter CA, Cottrill HM. A brief review of the management of platinum-resistant-platinum-refractory ovarian cancer. Med Oncol. 2017:34(6):103.

27. Dinca AL, Bîrla RD, Dinca VG, Marica C, Panaitescu E, Constantinoiu S. Prognostic factors in advanced ovarian cancer - a clinical trial. Chirurgia (Bucur). 2020;115(1):50-62.

28. Miyamoto M, Takano M, Goto T, Kato M, Sasaki N, Tsuda H, et al. Clear cell histology as a poor prognostic factor for advanced epithelial ovarian cancer: a single institutional case series through central pathologic review. J Gynecol Oncol. 2013;24(1):37-43.

29. du Bois A, Reuss A, Pujade-Lauraine E, Harter P, Ray-Coquard I, Pfistere J. Role of surgical outcome as prognostic factor in advanced epithelial ovarian cancer: a combined exploratory analysis of 3 prospectively randomized phase 3 multicenter trials. Cancer. 2009;115(6):1234-44.

30. Ghoneum A, Afify H, Salih Z, Kelly M, Said N. Role of tumor microenvironment in the pathobiology of ovarian cancer: insights and therapeutic opportunities. Cancer Med. 2018;7(10):5047-56.

31. Yang $L$, Wang $S$, Zhang $Q$, Pan $Y, L v Y, C$, Chen $X$, et al. Clinical significance of the immune microenvironment in ovarian cancer patients. Mol Omics. 2018;14(5):341-51.

32. Pinto MP, Balmaceda C, Bravo ML, Kato S, Villarroel A, Owen Gl, et al. Patient inflammatory status and CD4+/CD8+ intraepithelial tumor lymphocyte infiltration are predictors of outcomes in high-grade serous ovarian cancer. Gynecol Oncol. 2018;151(1):10-7.

\section{Publisher's Note}

Springer Nature remains neutral with regard to jurisdictional claims in published maps and institutional affiliations.

Ready to submit your research? Choose BMC and benefit from:

- fast, convenient online submission

- thorough peer review by experienced researchers in your field

- rapid publication on acceptance

- support for research data, including large and complex data types

- gold Open Access which fosters wider collaboration and increased citations

- maximum visibility for your research: over $100 \mathrm{M}$ website views per year

At BMC, research is always in progress.

Learn more biomedcentral.com/submissions 